\title{
Interoperable EHR Systems - Challenges, Standards and Solutions
}

\author{
Bernd Blobel ${ }^{1,2,3^{*}}$ \\ ${ }^{1}$ Medical Faculty, University of Regensburg, Germany \\ ${ }^{2}$ First Medical Faculty, Charles University Prague, Czech Republic \\ ${ }^{3}$ Health Competence Center Bavaria, Deggendorf Institute of Technology, Germany
}

\begin{abstract}
Background: Electronic Health Record Systems (EHRS) and Personal Health Record Systems (PHRS) are core components of infrastructure needed to run any health system. Objectives: As health systems undergo paradigm changes, EHRS and PHRS have to advance as well to meet the related interoperability challenges.
\end{abstract}

Methods: The paper discusses EHR types, implementations and standards, starting with different requirements specifications, systems and systems architectures, standards and solutions.

Results: Existing standards and specifications are compared with changing requirements, presenting weaknesses and defining the advancement of EHRS, architectures and related services, embedded in advanced infrastructure systems.

Conclusion: Future EHR systems are components in a layered architecture with open interfaces. The need of verifying data models at business domains level is specifically highlighted. Such approach is enabled by the ISO Interoperability Reference Architecture of a systemoriented, architecture-centric, ontology-based, policy- driven approach, meeting good modeling best practices.

\section{Keywords}

EHR systems; Architecture; Interoperability; Standards

\section{Correspondence to:}

Prof. Dr. habil. Bernd Blobel, FACMI, FACHI, FHL7, FEFMI, MIAHSI,

Medical Faculty, University of Regensburg, Germany.

E-mail: bernd.blobel@klinik.uni-regensburg.de
EJBI 2018; 14(2):10-19

Received: March 11, 2018

Accepted: March 20, 2018

Published: April 30, 2018

\section{Introduction}

The paper at hand is based on, and updates, publications reflecting activities on EHR systems, interoperability challenges and related standards, organized by, or jointly with, Jana Zvarova this Special Issue is dedicated to. That way, it acknowledges Jana Zvarova's engagement in the field. In this paper, her interdisciplinary focus and her priority given to both patients and health professionals are especially reflected.

Documentation and healthcare have been interrelated since the ancient times, thereby meeting the following functions: being a reminder for the carer, enabling communications between actors involved in care processes, serving as basis for education, acting as tool for developing the discipline. Later on, the legal perspective of documentation has been added. Besides observation, as well as performed or intended actions, documentation represents the context of the actors including knowledge, skills, experience, but also organizational and legal requirements. After Segen's Medical Dictionary, medical documentation consists of “operative notes, progress notes, physician orders, physician certification, physical therapy notes, emergency room records, or other notes and/or written documents; it may include ECG/EKG, tracings, images, X-rays, videotapes and other media" [1]. The terms medical chart, medical record or health record describe systematic documentation of a single patient's medical history [2], where the first two present an organization centric, and the third a cross-organizational perspective, thereby going beyond the original medical perspective by extending the considerations to social, environmental, prevention, lifestyle, and other implications. Electronic documentations add to those principles information and communication technologies (ICT) and related facilities.

Comprehensive and accurate information about the status and processes directly and indirectly related to the health of the subject of care must be provided and managed to enable safe, high quality, and efficient care services [3]. An early overview on the development levels of electronic healthcare records (EHCRs) has been provided by the US based Medical Record Institute [4] (Figure 1). 
According to ISO/TR 20514 Health informatics - Electronic health record - Definition, scope and context [5], an EHR is defined as "a repository of information regarding the health status of a subject of care in computer processable form". It provides the "ability to share patient health information between authorized users of the EHR and the primary role of the EHR in supporting continuing, efficient and quality integrated health care". The same ISO specification defines an EHR system as "system for recording, retrieving and manipulating information in electronic health records", or - adapted from the Institute of Medicine's Computer-based Patient Record System definition [6] "a set of components that form the mechanism by which electronic health records are created, used, stored and retrieved including people, data, rules and procedures, processing and storage devices, and communication and support" facilities.

There are different types of EHR depending on the viewpoint applied. From a logical and organizational perspective, we can distinguish centralized and de-centralized or distributed EHR systems. Regarding its focus, we define organization-centric Electronic Health Records (EHRs) on the one hand and Personal Health Records (PHRs) beyond the regulated medical perspective on the other hand. From the management perspective, we distinguish the professionally/organizationally moderated EHR from a personally moderated EHR where the subject of care controls the use by deciding privilege management and access control rules. Both are legal records. Contrary to those EHRs, PHRs cannot count for legal records, as they allow the subject of care to enter or delete any information. Regarding the time dimension, an EHR can represent an episode (EHR extract) or a life-long record (EHR service). We can distinguish EHR approaches according to the access model used (indirect via regional or national switch points vs. direct access to central EHR systems) or according to the implementation technology such as integrated system, federated system, or service oriented system. Because of their rational roots and driving factors, all those approaches have their right to exist at least temporarily. Therefore, they exist also practically in co-existence or concurrency. The aforementioned approaches develop continuously, thereby showing some convergence. The consequences of those EHR types for the interoperability level possible will be discussed in some more detail in Section 3.

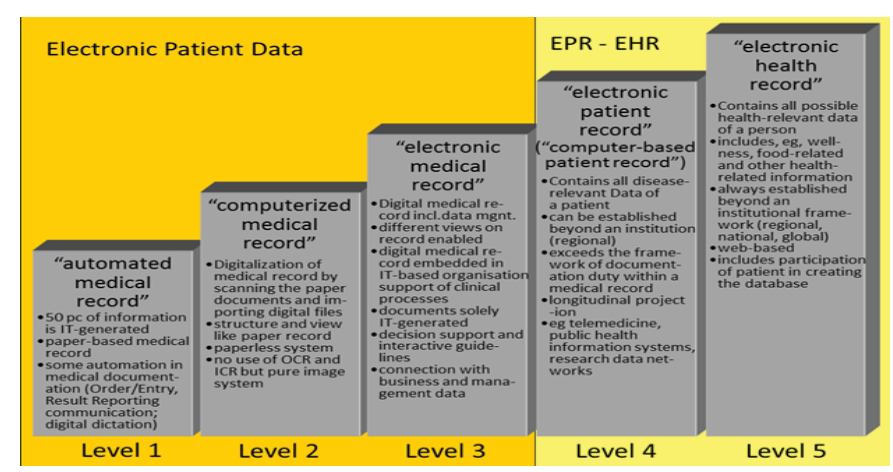

Figure 1. EHCR - Development Levels (Medical Record Institute, Newton, MA) [10].

\section{EHR Systems' Objectives and Requirements}

\subsection{Health Systems Paradigm Changes EHR Specifications and Solution Have to Meet}

For meeting the challenge to enhance quality and safety of patients' care as well as to improve efficiency and efficacy of care processes, changes regarding the organizational, methodological and technological paradigms for performing health service delivery are under way. Table 1 presents those changes at a high level.

EHR systems, enabled by corresponding architectural approaches, have to support those paradigm changes by undergoing the same transformation towards a highly complex, highly dynamic, multi-disciplinary/multi-domain advanced system in an intelligent, self-organizing way as the healthcare system does. Therefore, a systematic, formalized and adaptive approach is required to sharing and harmonizing ICT, domain, and personal ontologies, conditions and context at runtime.

For deploying EHR/PHR systems as core applications of health systems enabling cooperation for safe, high-quality and efficient care processes, certain content-related as well as functional requirements must be defined to be met by all involved systems. For that purpose, such requirements have been standardized or defined by institutions with sufficient authority. Examples of such requirements sets are the HL7/ISO standards EHR-S Functional Model and PHR-S Functional Model, but also the definitions of the Meaningful Use Program as well as the HIMSS Analytics Electronic Medical Record Adoption Model (EMRAM). The compliance of solutions with those requirements could be possibly certified to give vendors and providers, but also payers involved confidence in the adoption and maturity of those solutions. These requirements specifications are summarized below.

\subsection{HL7 Electronic Health Record System Functional Model (HL7 EHR-S FM)}

The HL7 EHR-Systems Functional Model (HL7 EHR-S FM) [7], also standardized at ISO as ISO/HL7 10781:2015 HL7 EHR-Systems Functional Model [8], provides a reference list of functions that may be present in an Electronic Health Record System (EHR-S) from a user perspective that allows managing and maintaining a health record for accomplishing various clinical, research and business purposes. Through the creation of Functional Profiles for care settings and realms, the EHR-S Functional Model enables a standardized description and common understanding of functions intended or available in a given healthcare setting (e.g. ambulatory care, behavioral health, 
Table 1. Paradigm changes impacting the transformation of health service delivery.

\begin{tabular}{|c|c|c|}
\hline Organizational & Methodological & Technological (Amount of Data) \\
\hline \begin{tabular}{|l} 
- Organization- \\
centric care \\
- Process- \\
controlled care \\
(DMP) \\
- Person-centric \\
care
\end{tabular} & $\begin{array}{l}\text { - General care addressing health problems (one } \\
\text { solution fits all) } \\
\text { - Phenomenological Approach } \\
\text { Dedicated care (stratification of population for } \\
\text { specific clinically relevant conditions) } \\
\text { - Evidence-Based Medicine } \\
\text { - Personalized, preventive, predictive and } \\
\text { participative care considering individual health } \\
\text { state, conditions and contexts, OMICS data, etc. } \\
\text { (stratification of population by risk profiles) } \\
\text { Systems Medicine, from art to multi- } \\
\text { disciplinary science, from elementary particle to } \\
\text { society } \\
\text { Cognitive care } \rightarrow \text { informed decision }\end{array}$ & $\begin{array}{ll}\text { - } & \text { Mainframe (KB) } \\
\text { - } & \text { Client/Server (MB) } \\
\text { - } & \text { Distributed systems, mobile } \\
\text { technologies, nano- and molecular } \\
\text { technologies, knowledge representation } \\
\text { \& -management, Artificial Intelligence, } \\
\text { Big Data \& Business Analytics, Cloud } \\
\text { Computing, Social Business, Cognitive } \\
\text { Computing (PB, YB) }\end{array}$ \\
\hline
\end{tabular}

intensive care, and cardiology, office practice in one country or primary care in another country). The Infoway Project Canadian EHR Blueprint introduced later some details as an example of a jurisdictional profile. It lists function identifier, function names and a related statement (meaning), but also conformance criteria (shall, should, may) and relationship to other functions. The HL7 EHR-S FM, R1, outlines important features and functions that should be contained in an EHR system. The HL7 EHR-S FM, R2, builds on Release 1.1 of the model, offering a more comprehensive set of functions and criteria.

\subsection{HL7 Personal Health Record System Functional Model (HL7 PHR-S FM)}

The HL7 Personal Health Record System Functional Model, Release 1 standard [9] specifies a standardized description and common understanding of features and functions necessary to create and effectively manage PHRs that help an individual maintain a longitudinal view of his or her health history. Personal Health Record information is expected to be sent, received, or exchanged from multiple systems, including: Electronic Health Record systems, insurer systems, payer systems, health information exchanges, public health systems, Internet-based health education sites, clinical trials systems, and/or collaborative care systems.

\subsection{ISO 18308 Requirements for an Electronic Health Record Architecture}

ISO 18308 [10] defines the set of requirements for an EHR architecture. The requirements are formulated to ensure that EHR systems are faithful to the needs of healthcare delivery, are clinically valid and reliable, are ethically sound, meet prevailing legal requirements, support good clinical practice and facilitate data analysis for a multitude of purposes. ISO 18308:2011 does not specify the full set of requirements that need to be met by an
EHR system for direct patient care or for other use cases, but the requirements defined by ISO 18308:2011 do contribute to the governance of EHR information within such systems.

\subsection{Electronic Medical Record Adoption Model}

The HIMSS Analytics Electronic Medical Record Adoption Model (EMRAM) [11] defines a methodology and algorithms to measure adoption and utilization of EMR functions, that way scoring the capabilities of EMR systems. The application of this model and the resulting assessment of EMR systems are not limited to the USA. Also hospitals in other countries around the world are assessed and get awarded the related EMRAM level [12]. EMRAM defines 8 levels of EMR adoption and maturity according to the inclusion of ancillary clinical systems such as pharmacy, laboratory, and radiology, PACS and non-DICOM image management, internal interoperability, security features, intelligent data management up to big data and analytics, cross-organizational interoperability and privacy. In some more details, the EMRAM levels are specified as follows:

- Level 0) No clinical ancillaries are installed.

- Level 1) The three basic ancillary clinical departmental systems (Laboratory IS, Pharmacy IS, Radiology/ Cardiology IS), but also the radiology/cardiology PACS and non-DICOM image management systems are installed.

- Level 2) A clinical data repository (CDR) enables full interoperability between the aforementioned units, supported by a controlled vocabulary and a clinical decision support (CDS) rule engine for rudimentary conflict checking; Furthermore, basic security services such as access control and usage management, encryption, mobile security, etc. are implemented. 
- Level 3) Nursing and allied health documentation (NAHD) is partially (50\%), in emergency departments fully, implemented in integrated in the CDR; Electronic medication administration record (eMAR) and role-based access control (RBAC) are installed.

- Level 4) Computerized practitioner order entry (CPOE) is partially $(50 \%)$, in emergency departments fully, implemented, supported by a clinical decision support (CDS) rule engine for rudimentary conflict checking; NAHD has grown from 50 to $90 \%$; Where publicly available, clinicians have access to national or regional databases and registries to support decision making; Nurses are supported by CDS capability related to evidence-based medicine protocols; Precautions for EMR downtime and network intrusion detection are implemented.

- Level 5) Full physician documentation with structured templates and discrete data is partially (50\%), in emergency departments fully, implemented in the hospital; Nurse order/task completion can be tracked and reported; Intrusion detection is extended to intrusion prevention; Mobile security is implemented, including the opportunity to wipe remotely data from lost/stolen devices.

- Level 6) Comprehensive process management for administering medications, blood products, and human milk is partially (50\%), in emergency departments fully, implemented; Integration of eMAR with CPOE, Pharmacy IS and Laboratory IS improves safe point-ofcare processes and results; Process management is CDS supported; Mobile/portable device security is applied to user-owned devices, that way properly managing BYOD (bring your own device) policies.

- $\quad$ Level 7) Disbanding paper charts and integrating all data modalities completely in the EMR system by supporting all aforementioned capabilities including data governance, disaster recovery, security and privacy; Deployment of data warehousing to analyze clinical data patterns for improving care quality, patient safety, and care delivery efficiency; Health information exchange (HIE) across the healthcare ecosystem, using standardized health information exchange protocols such as CCD or FHIR resources [13]; Continuity of care is realized throughout the hospital.

\subsection{Meaningful Use}

The US Health Information Technology for Economic and Clinical Health (HITECH) Act as part of the American Recovery and Reinvestment Act was signed into law in 2009 to promote the adoption of electronic health records through meaningful use. For improving quality and efficiency of care delivery as well as enhancing coordination between providers, but also privacy and security of personal information and the engagement of patients in their own health through health information and health information exchange (HIE), the US Department for Health and Human Services (HHS) has established an EHR Incentive and Certification Program managed by the Centers for Medicare \& Medicaid Services (CMS). It defines requirements and objectives for EHR systems of eligible providers, both professionals and hospitals, to benefit from that program.

\section{The EHR Incentive Programs consist of three stages} [14]:

- $\quad$ Stage 1 set the foundation for the EHR Incentive Programs by establishing requirements for the electronic capture of clinical data, including providing patients with electronic copies of health information. In detail, the following functionalities are required: Implementation of drug-drug and drug-allergy interaction checks; maintaining problem, medication and medication allergy lists; recording of specific demographics, vital signs and children's smoking information; implementation of CDS rules; reporting quality measures; establishing basic security and privacy services.

- $\quad$ Stage 2 expanded upon the Stage 1 criteria with a focus on advancing clinical processes and ensuring that the meaningful use of EHRs supports the aims and priorities of the National Quality Strategy. Stage 2 criteria encouraged the use of certified EHR technology (CEHRT) for continuous quality improvement at the point of care and the exchange of information in the most structured format possible. Addressed functionalities are: Implementation of drug formulary checks; recording of advanced directives for elderly as well as structured clinical lab-test results; providing patient-specific education resources; performing medication reconciliation and sharing patient summaries with units collaborating in those patients' care; sharing related information with public registries and public health agencies.

- In October 2015, CMS released a final rule that modified Stage 2 to ease reporting requirements and to align with other quality reporting programs, such as the Medicare Access and CHIP Reauthorization Act (MACRA) and the Quality Payment Program. The latter offers two streams: Advanced Alternative Payment Models (APMs) or the Meritbased Incentive Payment System (MIPS). This final rule also established Stage 3 in 2017 and beyond, which focuses on using CEHRT to improve health outcomes. In detail, EHR systems shall provide: Patients' electronic access to their information; advanced reporting schemes to registries and authorities; security risk analysis; comprehensive HIE with collaborating healthcare establishments.

\section{EHR Systems ${ }^{6}$ Interoperability}

Communicating and cooperating actors involved in a business case have to share data related to the business 
process, interpret them to derive the corresponding information to be used for performing the actions needed to jointly meet the business objectives. All three steps of the so-called information cycle [15] are depending on appropriate knowledge and skills available at the actors.

With the advent and further advancement of IT in health, the term interoperability has evolved very much. In its Standards Glossary, IEEE defines interoperability as "ability of a system or a product to work with other systems or products without special effort on the part of the customer" [16]. HIMSS defined 2013 interoperability in healthcare as "the ability of different information technology systems and software applications to communicate, exchange data, and use the information that has been exchanged", thereby distinguishing the three interoperability levels a) foundational, b) structural and c) semantic interoperability. Some years ago the author introduced a more comprehensive definition of interoperability beyond technologies: "Interoperability describes motivation, willingness, ability, and capability to cooperate for achieving common goals or business objectives. It requires knowledge, abilities and skills, shared and adapted a-priori or dynamically at runtime." [17]. Thereby he refined the Knowledge Interoperability level of his originally introduced interoperability levels Data Interoperability, Information Interoperability, and Knowledge Interoperability as presented in Table 2, distinguishing both the information and the organization perspective.

\subsection{EHR Systems Architectures}

According to IEEE 1471 [18], a system as a collection of components organized to accomplish a specific function or set of functions can be distinguished from its environment, the system's context, which may influence that system by setting constraints. Systems can be decomposed to subsystems or composed to supersystems in a recursive way. A system has one or more stakeholders having special interests in, or concerns to, that system. For meeting the stakeholders' intended objectives of the system, it has to fulfill one or more missions in its environment, also named business objectives.

Every system has an architecture, i.e. according to IEEE 1471, the fundamental organization of a system embodied in its components, their relationships to each other and to the environment and the principles guiding its design and evolution. The architectural description of the system, formally representing the intended artefacts of the system, is organized in architectural views representing a related set of concerns of particular stakeholders. Views are constructed and managed according to viewpoint specifications with their specific language and techniques to represent the view's knowledge (including notations, modeling methods, models, product types, etc.).

ISO/TS 18308 defines EHR architecture as "generic structural components from which all EHRs are built, defined in terms of an information model" [10]. In extension to that definition, ISO TR 20514 states in the EHR Architecture Definition Note "A more descriptive informal definition of an EHRA is that of a model of the generic features necessary in any electronic healthcare record in order that the record may be communicable, complete, a useful and effective ethico-legal record of care and may retain integrity across systems, countries and time...."

From a representational perspective of specifying and implementing EHR architectures, we classify a) data approach (data representation), b) concepts approach (concept/knowledge representation), and c) process/service approach (business_process/service representation). Those three streams partially have their roots in existing systems, in traditional thoughts and methodologies as well as in specific domain-languages and modelling languages. Related to the representational perspective is the modeling perspective. Here we can distinguish a) component-oriented single model approach [19], b) component-oriented dual model approach [20] and c) multi-model approach of componentoriented services $[21,22]$. The first two approaches pursue data integration, embedding concepts into structures in the case of the single model approach, or specifying and implementing them using concept models (archetypes) and their implementable object models in the case of the dual model approach. They may be imported into applications to enable functionalities such as workflow concepts and alert mechanisms that are derived from the available data. The third approach realizes functional integration, i.e. interoperability due to the architectural paradigm that is deployed. Those three approaches require different levels of knowledge sharing. The first is based on a-priori distributed knowledge, the second shares data and corresponding concepts, and the third provides facilities to deploy the underlying knowledge.

Regarding the protocol deployed for communication and cooperation, there is a communication focus (message), a document focus (clinical document), and a business process focus (application). Artefacts implementing EHR systems interoperability have evolved during the last 25 years from structured messaging (e.g. EDI, HL7 messaging) over sharing concepts (e.g. openEHR Archetypes ${ }^{1}$, EN/ISO 13940 ContSys concepts [23]) - both representing the data/information exchange paradigm - to cooperation at application level (e.g. Web services, FHIR resources). Nevertheless, all those standards-based interoperability approaches are restricted to computer-to-computer communication, representing information according to the domain independent ISO/IEC 10746 Information technology - Open Distributed Processing - Reference Model or to domain-specific information models

${ }^{1}$ An archetype is a model of a clinical or other domain-specific concept which
defines the structure and business rules of the concept; computable expression
of a domain-level concept in the form of structured constraint statements, of a domain-level concept in the form of structured constraint
based on some reference information model (ISO/TR20514) [5]. 
Table 2. Interoperability levels

\begin{tabular}{lll}
\hline Information Perspective & & Organization Perspective \\
\hline Interoperability Level & Instances & Interoperability Level \\
\hline Technical & Technical plug \& play, signal \& protocol compatibility & Light-weight interactions \\
Structural & Simple EDI, envelopes & Data sharing
\end{tabular}

\section{Syntactic \\ Messages and clinical documents with agreed vocabulary}

Semantic

Organization/Service

Knowledge based

Skills based
Advanced messaging with common information models and terminologies

Common business process
Information sharing

Knowledge sharing at IT concept level Coordination

Knowledge sharing at business concept level Agreed cooperation

Knowledge sharing at domain level Cross-domain cooperation

Knowledge sharing in individual context Moderated end-user collaboration

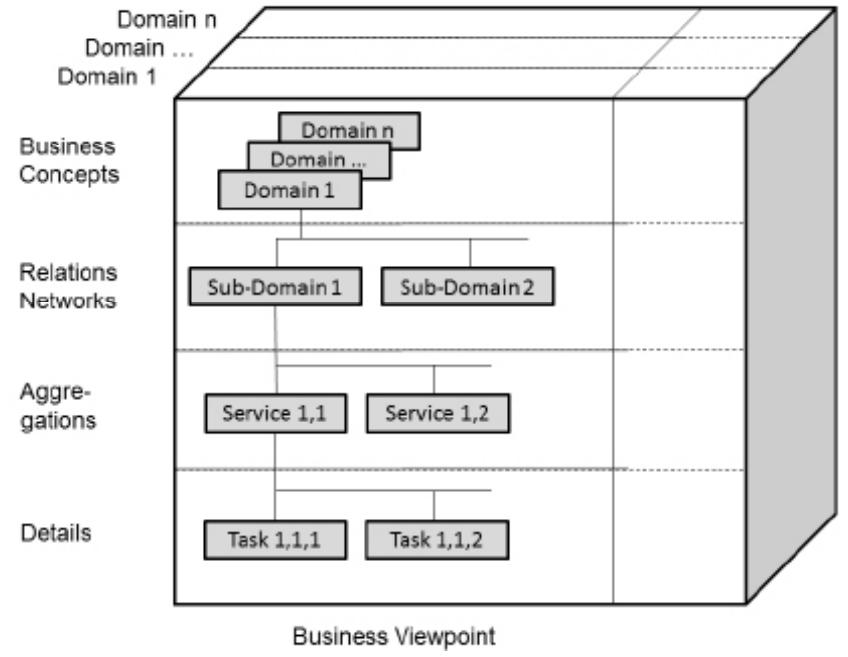

Figure 2. ISO interoperability reference architecture model granularity levels.

such as ISO/HL7 21731 Health informatics - HL7 Version 3 Reference Information Model. [24].

\subsection{The ISO Interoperability Reference Architecture}

The ISO Interoperability Reference Architecture is an abstract domain-independent representation of systems using Universal Type Theory and corresponding logics. The mathematical concept representation in combination with systems engineering methodologies allows representing any system architecturally (i.e. the system's components, their functions and internal as well as external relations) by generically describing its composition/ decomposition as well as the aspects (domains) of the system relevant in a specific context (e.g. business case). For correctly and formally representing the concepts and relations of the domain-

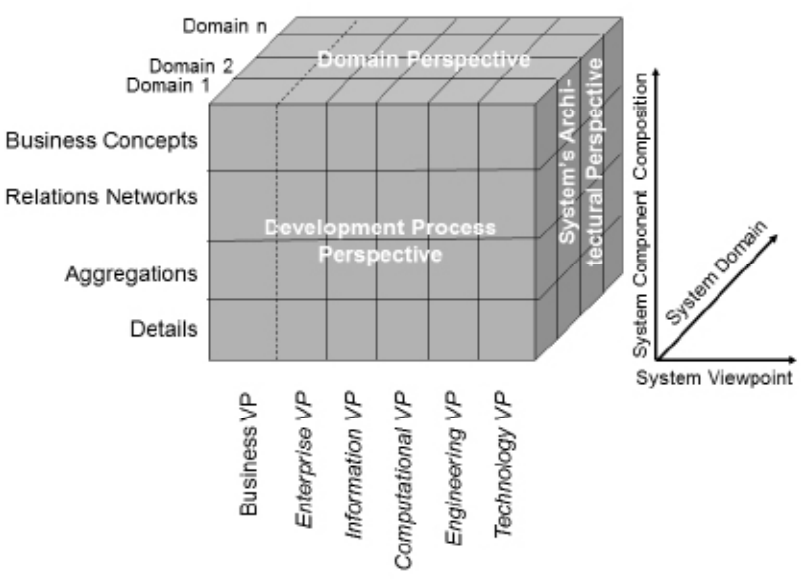

Figure 3. The interoperability reference architecture model.

specific subsystems involved in that business case, those subsystems are represented by their corresponding approved domain ontologies, resulting in a system-theoretical, architecture-centric, top-level ontology driven approach [25]. The reference architecture model can be used recursively, so representing, e.g., the real-world systems' continuum from elementary particles to the universe (Figure 2).

By combining that Business Viewpoint granularity levels model with ISO/IEC 10746, the Interoperability Reference Architecture Model (introduced in the nineties by the author as Generic Component Model - GCM) as well as the applicable rules - the Interoperability Reference Architecture Framework - (also known as GCM Framework) is completed (Figure 3) [17]. 
This Interoperability Reference Architecture Model allows consistently transforming and interrelating any domain-specific subsystem's structure and behavior (e.g. domain-specific standards and specifications) by ontologically representing its concepts and relationships at the real world system component's level of granularity. In other words, the domain-specific subsystem (e.g. a domain-specific standard or specification) is re-engineered using the Interoperability Reference Architecture Model, by that way providing a standardized interface to that specification. The ISO Interoperability Reference Architecture has been included in ISO 13606 Health informatics - EHR communication [19] and demonstrates the architecturally correct representation of ISO 13606 based EHR systems and their integration in security and privacy specifications. It supports ontology harmonization or knowledge harmonization to enable interoperability between existing systems, standards and solutions of any level of complexity without the demand for continuously adapting/revising those specifications. In summary the ISO Interoperability Reference Architecture including the GCM Framework principles can be used.

- for analyzing, designing, and implementing EHR systems and underlying architectural models characterized by their components, functionalities, and relationships,

- $\quad$ for defining and realizing migration strategies, but also

- $\quad$ for evaluation, gap analysis and roadmap definition in standards development.

More information on harmonizing existing EHR systems specifications is provided, e.g., in [26].

\section{$4 \quad$ EHR Systems Standards and Solutions}

Longitudinal sharable EHR must support collaborative care processes, properly and transparently representing the knowledge of all domains contributing to the care process in a way enabling correct, informed and consistent care decisions. The justification of correctness and consistency in the framework of the disciplines' knowledge can finally only be provided at the real world scenario, as explained in some details in [27]. EHR have to be open, scalable, flexible, portable, distributed, standardconformant, interoperable at an appropriate level, business process responsive, service-oriented, user-accepted, applicable to any media, trustworthy and lawful. Therefore, the following architectural paradigms have to be met: distribution; componentorientation; a model-driven and service-oriented design taking into account concepts, context, and knowledge; comprehensive business modeling; separation of computation-independent, platform-independent, and platform-specific modeling (thus separating the functional and the logical from the technological view); agreed reference terminologies and ontologies; a unified development process; and advanced security and privacy services embedded in the architecture. Examples of EHR standards, projects and solutions are: HL7 Clinical Documents Architecture (CDA) documents, ASTM E 2369 "Standard Specification for
Continuity of Care Record (CCR)", HL7 Continuity of Care Documents (CCD - implementation of CCR in CDA), or Archetypes defined by the openEHR Foundation and in ISO/ EN 13606 "Health informatics - EHR communications", but also related IHE profiles or DICOM specifications. Those and more examples have been presented in some details, evaluated and referenced e.g., in [22, 24]. Most of them meet just very few of the aforementioned characteristics.

\section{Discussion}

\subsection{Critical Views on EHR Systems, Standards, Programs and Solutions}

Despite all specifications, standards and quite expensive programs, existing EHR approaches - even if certain aforementioned characteristics have been considered in the EHR system design and more or less appropriately implemented - do not accomplish the end users' requirements and expectations. Sue Bowman [28] has highlighted problems with improper EHR specifications and implementations. Many of them show design flaws partially caused by the growing complexity of systems, so not fully meeting the promise of improving care quality, patient safety, and process efficiency. They miss, for example, the needed flexibility and adaptability, but depend on the context and organization they have been designed for. Frequently, the workflow assumed is not the way health professionals actually practice, provoking the deployment of workarounds impacting patient safety. Another risk deals with inconsistencies between structured and free-text data, but also with lack of transparency of ICT processes and actions. In that context, automated processes, e.g. automated data capturing, when inappropriately deployed, can lead to erroneous documentation. That way, inaccurate or outdated information could be entered, especially when using templates. Another challenge is the proper design and implementation of clinical decision support systems, sometimes leading to the so called automation bias. Partially, those problems could be overcome by certifying EHR systems as done in the Meaningful Use (MU) context. However, a multi-center study analyzing experiences in the MU initiative context has demonstrated a series of weaknesses for meeting the overarching objective of care quality, patient safety and care process efficiency [29]. Here, deficiencies regarding quality and accuracy of medication lists and medication reconciliation, but also of problem list and allergy list have to be mentioned. Another critical point is constraining EHR systems functionalities and innovations on the defined incentives, thereby ignoring serious clinical interest in wider developments including the missing consideration and integration of external data describing the patient's behavior and context. Furthermore, clinicians requested better CDS by carefully balancing sensitivity vs. specificity as well as a stronger focus on specific population such as children, developing child-specific standards and norms, and citizens 
in rural areas, establishing the educational and technological infrastructure needed. Finally, more flexibility in the application and interpretation of the MU program is recommended.

A summary of all aforementioned requirements has been provided by the AMIA EHR-2020 Task Force on the Status and Future Directions of EHRs [30]. Here, five areas of development and improvement have been defined: simplification and speeding up documentation, refocusing regulation, increasing transparency and streamlining certification, fostering innovation, supporting person-centered care delivery.

Because of high administrative burdens, costs, and partially insufficient outcome, parts of the US EHR Incentive Program will be redesigned, for example promoting the newest US initiative MyHealthEData [31].

\subsection{Future Directions}

For guaranteeing data quality, integrity and correctness, EHR systems in general, but especially when moving to PHR systems, need implementing a quality management system and governance management, but also solving legal, accessibility and usability issues. The infrastructural services necessary in the sense of flexible and adaptive cognitive systems shall comprise context monitoring, text analytics (automated text analysis), natural language processing (NLP), etc.

For properly integrating clinical data, semantic mapping, a data lake infrastructure supporting multiple file formats for data intake, but also the aforementioned service of text analytics and NLP are inevitable for converting unstructured into structured formats.

Traditionally, EHRs are first of all designed to provide actors involved the information they need to participate in the care process. They collect data within a specific workflow, not supporting data utilization outside that workflow including the deployment of transactional data. When looking for future requirements to meet the healthcare transformation challenge presented in Table 1, it is impossible to provide EHR systems for functionalities such as artificial intelligence and machine learning, real-time analytics on transactional data, dynamic binding to data from any system, making data management and services available to any system. Therefore an additional layer on top of the EHR system must be introduced, interconnecting with the other layers via open APIs like Health Catalyst's Data Operating System (DOS). That way, the data operating ecosystem creates real-time insight to meet the aforementioned healthcare transformation challenges of quality care, patient safety and care process efficiency and provides them back to the healthcare ecosystem with its core component EHR system. In detail, following services have to be provided [32]:

- Real-time analysis of data from multiple data sources to monitor patients;

- $\quad$ Patient dashboards to monitor a patient's overall health and key improvement measures;
- $\quad$ Early detection of at-risk patients;

- Discovery of population health trends;

- Custom rules based on specific data triggers, which can be used to create follow-ups if certain patient health indicators are met;

- Performance insights on staff productivity;

- Data mining capabilities to discover insights on different patient conditions; and

- Data analysis to determine the most effective treatment plans for patients.

An early and far simpler approach to such a layered EHR system architecture has been defined and implemented with the Canada Infoway EHR Solution Infostructure [33].

Based on the current developments, the adoption of new and emerging technologies such as artificial intelligence and machine learning, so enabling the aforementioned flexibility and adaptability and accommodating any thinkable use case could be pushed by Google's open source Cloud Healthcare API, introducing a second tier above the EHR system. Google's API is based on HL7's FHIR API. As alternative to Google's Cloud Healthcare initiative, Amazon Web Services (AWS) or Microsoft's Azure have to be mentioned.

Combining the aforementioned Canada Infoway EHR Solution Infostructure and its German adoption the author was responsibly involved in with the Cancer Care Ontario Enterprise Architecture [34] and elements of IBM's Clinical Decision Intelligence approach considering the patient's environmental and contextual conditions by cognitive computing [35], the essential elements of a future EHR System Enterprise Architecture can be derived as shown in in Figure 4.

Following those advanced approaches increases the importance of data governance, but also of security, privacy and trust in the ecosystem.

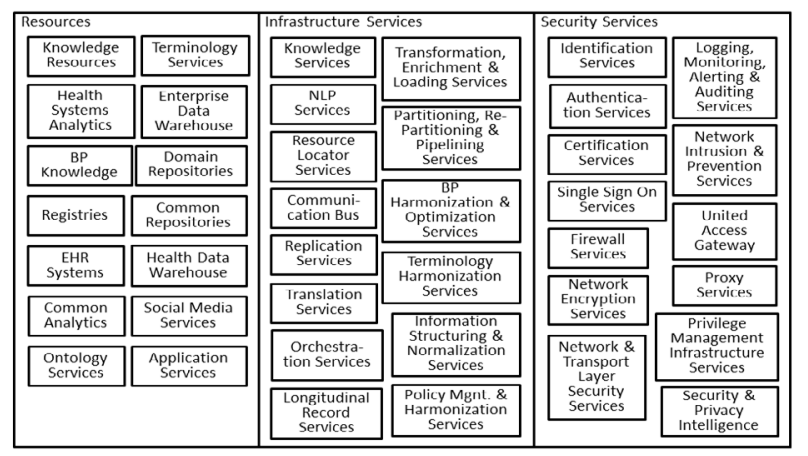

Figure 4. Essential components of a future EHR system enterprise architecture. 


\section{Conclusion}

Over the 50 years of developing EMRs/EHRs since the pioneering developments of Lawrence (Larry) Weed, resulting in a series of international or national standards and projects, the main focus was put on the EHR structure, but less on EHR functionality. In consequence, there was a strong and almost "religious" competition between the different EHR types and EHR approaches presented in the paper. Hopefully it became clear that the focus has to change to services, implemented as data services, but justified through the disciplines creating those data as description and representation of those domains' concepts.

That way, EHR systems will master the ongoing healthcare transformation according to the health systems' organizational, methodological and technological paradigm changes, thereby meeting requirements and expectations of domain-specific stakeholders including the patients.

Despite all advancements in data-driven solutions, any project has to start in modeling the business domain(s), where the stakeholders define requirements, base concepts and relations, etc., according to the modeling best practice principles [36, 37, $38,39,40]$. Only in the real world domains, correctness and consistency of concepts and rules, i.e. ontologies, can be justified. Otherwise we face the problem of many IT and informatics projects that the designed map is perfect, just the landscape represented is wrong.

\section{Acknowledgement}

The author is indebted to thank his colleagues from standards bodies and institutions such as ISO, CEN, HL7, the EuroRec Institute as well as related projects like GEHR/ openEHR and Q-REC for kind cooperation and support. Furthermore, he likes to thank Arie Hasman and Dipak Kalra for critical reading and editorial support.

\section{References}

[1] The Free Dictionary. Medical Documentation. Farlex, Inc.: Huntingdon Valley; 2012. https://medical-dictionary. thefreedictionary.com/medical+documentation

[2] Wikipedia. Medical Record. https://en.wikipedia.org/wiki/ Medical_record

[3] Blobel B. Analysis, Design and Implementation of Secure and Interoperable Distributed Health Information Systems. Amsterdam: IOS Press; 2002.

[4] Medical Record Institute, Newton, M A, USA. http://www. medrecinst.com

[5] International Organization of Standardization. ISO/TR 20514:2005 Health informatics -Electronic health recordDefinition, scope and context. Geneva: ISO; 2005.

[6] Dick RS, Steen EB, Detmer DE. (eds.) The Computer-Based Patient Record: An Essential Technology for Health Care,
US National Academy of Sciences, Institute of Medicine, Washington DC: National academy press ; 1991.

[7] HL7 International Inc. HL7 EHR-System Functional Model, R2. Ann Arbor: HL7; May 2017.

[8] International Organization of Standardization. ISO/ HL7 10781:2015 HL7 EHR- Systems Functional Model. Geneva: ISO; 2015.

[9] HL7 International Inc. HL7 Personal Health Record System Functional Model, Release 1. Ann Arbor: HL7; May 2014.

[10] International Organization of Standardization. ISO 18308:2011 Health informatics - Requirements for an electronic health record architecture. Geneva: ISO; 2000.

[11] HIMSS Analytics. EMRAM: A strategic roadmap for effective EMR adoption and maturity. Chicago: HIUMSS Analytics; 2018. http://www.himssanalytics.org/emram

[12] Inés Burton. Aaplesion Diakonieklinikum Rotenburg erreicht HIMSS Analytics EMRAM Stufe 6. HIMSS Europe; 2018.

[13] HL7 International Inc. Fast Healthcare Interoperability Resources (FHIR) V. 1.0.2. Ann Arbor: HL7; 2015. www. hl7.org/fhir

[14] Center for Medicare \& Medicaid Services. Electronic Health Records (EHR) Incentive Programs. https:// www.cms.gov/Regulations-and-Guidance/Legislation/ EHRIncentivePrograms/index.html

[15] Van Bemmel J., Musen M. (eds.) Handbook of Medical Informatics. Heidelberg: Springer; 2002

[16] IEEE Standards University. Standards Glossary. Piscataway: IEEE SA; 2016.

[17] Blobel B. Architectural approach to eHealth for enabling paradigm changes in health. Methods Inf Med. 2010; 49: 123-134.

[18] IEEE Standards Association. ANSI/IEEE 1471-2000 - IEEE Recommended Practice for Architectural Description for Software-Intensive Systems. Piscataway: IEEE SA; 2000.

[19] International Organization for Standardization. ISO 13606:2018 Health informatics- EHR communication. Geneva: ISO; 2018.

[20] Beale T. A Model Universe for Health Information Standards; 2003. http://www.deepthought.com.au

[21] Blobel B. Application of the component paradigm for analysis and design of advanced health system architectures. Int J Med Inf. 2000; 60: 281-301.

[22] Blobel B. Advanced and secure architectural EHR approaches. Int J Med Inf. 2006; 75: 185-190. 
[23] International Organization for Standardization. ISO 13940:2015 Health informatics - System of concepts to support continuity of care. Geneva: ISO; 2015.

[24] Blobel B, Pharow P. Analysis and Evaluation of EHR Approaches. Methods Inf Med. 2009; 48: 162-169.

[25] Blobel B. Knowledge Representation and Management Enabling Intelligent Interoperability - Principles and Standards. Stud Health Technol Inform. 2013; 186: 3-21.

[26] Blobel B. Standardization for Mastering Healthcare Transformation - Challenges and Solutions. EJBI. 2017; 13: 9-15.

[27] Blobel B, Goossen W, Brochhausen M. Clinical modeling - A critical analysis. Int J Med Inf. 2014; 83: 57-69.

[28] Bowman S. Impact of Electronic Health Record Systems on Information Integrity: Quality and Safety Implications. Perspect Health Inf Manag. 2013 Fall; 10(Fall): 1c.

[29] Slight SP, Berner ES, Galanter W, Huff S, Lambert BL, Lannon C, et al. Meaningful Use of Electronic Health Records: Experiences from the Field and Future Opportunities. JMIR Med Inform. 2015; 3: e30.

[30] Payne TH, Corley S, Cullen TA, Gandhi TK, Harrington L, Kuperman GJ, et al. Report of the AMIA EHR-2020 Task Force on the status and future direction of EHRs. J Am Med Inform Assoc. 2015; 22:1102-1110.

[31] Centers for Medicare \& Medicaid Services. Trump Administration Announces MyHealthEData Initiative at HIMSS18. https://www.cms.gov/Newsroom/MediaReleaseDatabase/ Fact-sheets/2018-Fact-sheets-items/2018-03-06.html
[32] Chouffani R. Transition to value-based care supported by data analytics. TechTarget, SearchHealthIT. 2017. http://searchhealthit.techtarget.com/tip/Transition-tovalue-based-care-supported-by-data-analytics

[33] Canada Health Infoway Inc: EHR-S Blueprint, Version 2, 15 April 2006. https://www.infoway-inforoute.ca

[34] Cancer Care Ontario. Cancer Care Ontario Enterprise Architecture Blueprint 2011-15. GOV-CCO-6150, March 2014. https://archive.cancercare.on.ca/about/ architecture/

[35] Wang XS, Nayda L, Dettinger R. Infrastructure for a clinical-decision-intelligence system. IBM Syst J. 2007; 46: 151-169.

[36] Lankhorst M, Dietz J, Erik P, Tribolet J. (eds.) Enterprise Architecture at Work. The Enterprise Engineering Series. Berlin Heidelberg: Springer- Verlag; 2009.

[37] Rebstock M, Fengel J, Paulheim H. Ontologies-Based Business Integration. Berlin Heidelberg: SpringerVerlag; 2008.

[38] Seiner RS. Data Modeling is a Form of Data Governance. IDERA. 2017.

[39] Hoberman S, Burbank D, Bradley C. Data Modeling for the Business: A Handbook for Aligning the Business with IT using High-Level Data Models. New Jersey: Technics Publications; 2009.

[40] Krogstie J. Business Information Systems Utilizing the Future Internet. LNBIP. 2011; 90: 1-18. 